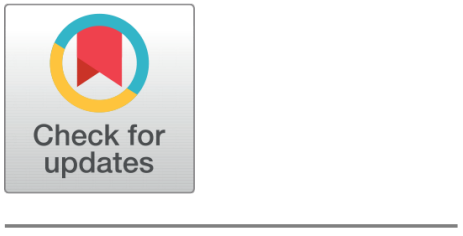

OPEN ACCESS

Received: 07.11.2020

Accepted: 22.12 .2020

Published: 22.02 .2021

Citation: Susanth SG (2021) Observations of long period HF Doppler oscillations over the dip equatorial ionosphere in the Indian longitudinal sector. Indian Journal of Science and Technology 14(6):

541-546. https://doi.org/

10.17485/IJST/v14i6.2023

* Corresponding author.

Tel: +91 9995060237

sgsusanthsg@gmail.com

Funding: None

Competing Interests: None

Copyright: (c) 2021 Susanth. This is an open access article distributed under the terms of the Creative Commons Attribution License, which permits unrestricted use, distribution, and reproduction in any medium, provided the original author and source are credited.

Published By Indian Society for Education and Environment (iSee)

ISSN

Print: 0974-6846

Electronic: 0974-5645

\section{Observations of long period HF Doppler oscillations over the dip equatorial ionosphere in the Indian longitudinal sector}

\author{
S G Susanth ${ }^{1 *}$ \\ 1 Assistant Professor, Department of Applied Science and Humanities, Lourdes Matha \\ College of Science and Technology, Trivandrum, 695574, India. Tel.: +919995060237
}

\begin{abstract}
Background/ Objective: The signatures of long period High Frequency Doppler (HFD) oscillations over a dip equatorial station, Trivandrum $\left(8.33^{\circ} \mathrm{N}\right.$, $77^{\circ} \mathrm{E}$, dip $0.4^{\circ} \mathrm{N}$ ) in the Indian longitudinal sector have been investigated. Methods: High cadence vertical plasma drift measurements obtained using a Multi frequency HF Doppler Radar over Trivandrum along with ground based magnetometer observations over several latitudes/longitudes have been used to address this very aspect. Findings: It has been observed that long period ( 15 min.) oscillations of high latitude origin can instantaneously reflect over the equatorial latitudes in the form of coherent HFD oscillations. The study is very important as it provided a unique opportunity not only to understand various characteristics of HFD oscillations, but also expected to have significant implications in the understanding of equatorial ionospheric variability in context of solar wind-magnetosphere-ionosphere coupling. Novelty: In a complementary point of view, the study also highlights the potential of HF Doppler measurements to capture such transient events originating from interplanetary medium with an unprecedented temporal resolution.
\end{abstract}

Keywords: HF Doppler radar; equatorial ionosphere; HF Doppler oscillations

\section{Introduction}

HF Doppler radar over Trivandrum has provided significant results concerning the equatorial electrodynamics and associated phenomena. Although radar observations have been mostly used to address the Pre-reversal enhancement and its behavior during evening hours, they have been widely used to address the morning time electrodynamical drift too. In one of the earliest studies, the data have been effectively utilized to understand the characteristics of meridional wind over Trivandrum and has been compared with a co-located ionosonde too. Furthermore, in the past, attempts have also been made to understand the evening time characteristics of the velocity vortex over Trivandrum. The importance of chemical corrections in the observed vertical plasma drifts measurements and their quantitative estimates during evening and morning hours have also been reported. Recently, the important scientific results 
obtained using the HF Doppler radar over Trivandrum has also been reviewed elsewhere ${ }^{(1)}$.

HF Doppler (HFD) oscillations are basically the periodic fluctuations in the Doppler frequency observed over mid/low or equatorial latitudes in coherent with the geomagnetic disturbances of high latitude origin. They are very important as they provide unique opportunity to investigate various aspects of the high latitude-low latitude coupling in addition to the solar wind-magnetosphere ionospheric coupling. High latitude magnetic disturbances can manifest as the correlated fluctuations in HF Doppler signals due to either the (i) electric field generated in the E region influencing the F-region as ExB drift or (ii) motion of F-region due to magnetohydrodynamic (MHD) wave ${ }^{(2)}$. These HFD oscillations are generally observed during various occasions such as geomagnetic storms, pulsation events, sudden storm commencements (SC) etc. Although they are initially interpreted as the phase changes in the path of radio signal caused by the ionospheric vertical motion, they are believed to have association with pulsation continuous $(\mathrm{Pc})$ and pulsation irregular (Pi) events. In fact, historically, they have been observed in connection with DP2 as well as sub storm events too ${ }^{(3)}$.

In general, it is understood that most of the HFD oscillations at the mid/low latitude associated with geomagnetic pulsations are in the Pc3 4 (10-150 s) or Pi2 (45-150 s) range. During extreme magnetic disturbed periods, effects of pc5 pulsations (150-600 s) in the form of HFD over equatorial/low latitudes have also been noticed. Such pc5 HFD have been attributed to either a manifestation of the compression mechanism acting on the low-latitude ionosphere ${ }^{(4)}$ or to the Pc5-related electric field originating in the high-latitude ionosphere and extending to the equator ${ }^{(5)}$. In the similar line, penetration of SC related geomagnetic fluctuations having periods 0.5 to a few hours, known as DP2 fluctuations have also been observed over equatorial/low latitudes. This has been explained due to the instantaneous transmission of TM0 mode through the earthionosphere waveguide ${ }^{(6)}$. Although long period (5-15 min.) pulsations are common over auroral latitudes, their counterpart HFD oscillations over equatorial/low latitudes have not been properly understood so far, mainly due to the paucity of observations. In this context, in the present study, an attempt is made to understand the effects of long period oscillations ( $15 \mathrm{~min}$ ) of high latitude origin in the HF Doppler signals over the dip equator. We present the observations on 14 August 2003 and 15 May 2005, obtained using a HF Doppler radar over the geomagnetic dip equatorial station, Trivandrum $\left(0.8^{\circ} \mathrm{N}\right.$, $\left.77 \mathrm{E}, 0.5^{\circ} \mathrm{dip}\right)$ in the Indian longitudinal sector. The plausible mechanism for these observations is explored in detail.

\section{Experiment}

A multi frequency HF Doppler radar system suitable for the ionospheric F-region vertical drift studies has been installed at the magnetic equatorial station, Trivandrum $\left(8.33^{\circ} \mathrm{N}, 77^{\circ} \mathrm{E}, \operatorname{dip} 0.4^{\circ} \mathrm{N}\right)$, Kerala, India. The HF Doppler radar is a monostatic pulsed coherent system capable of operation at three frequencies, viz. $2.5 \mathrm{MHz}, 3.5 \mathrm{MHz}$ and $4.5 \mathrm{MHz}{ }^{(7)}$. The system can work both in single frequency and in multifrequency modes. For the present study, we have used the observations using $3.5 \mathrm{MHz}$. The radar was designed, assembled and installed at the University of Kerala, Trivandrum, India ${ }^{(7)}$. The transmitter, receivers and antenna systems were procured form M/s. Tomco Electronics, Australia. For the vertical drift data, the time resolution for single frequency operation is one minute and it can be programmed to get higher resolution. For multi-frequency operation, there is a sequential switching scheme such that the system is operated in one frequency for one minute and after a delay of two minutes the next frequency is operated for one minute and so on. The two minutes delay in between data collections for two frequencies is required for changing the frequency settings. Thus, we are getting nearly simultaneous vertical drift data at two different heights with a time interval of two minutes. It is assumed that the ionospheric conditions change little within these two minutes ${ }^{(8)}$.

The F region vertical plasma drift, which is actually due to the horizontal electric field, is measured from the Doppler shifted reflected echoes. The recorded data from Doppler radar are Fourier analyzed for getting the prominent Doppler frequency $\left(\mathrm{f}_{D}\right)$ corresponding to up or down F-region movement. The vertical plasma drift velocity is calculated using the relation, $\mathrm{Vz}=$ $-\left(f_{D} \times \lambda\right) / 2$, where $\lambda$ is the sounding wavelength ${ }^{(9,10)}$. This reflection height is measured from the delay of time between the transmitted signal and the echo for each operating frequency, which has a resolution of $1.5 \mathrm{~km}$. The radar is capable of recording vertical drift data in the evening-to-morning sector between 17:00 hr LT and 08:00 hr LT with 1 minute data resolution. However, the measured drift velocity has to be corrected as it includes the contribution due to chemical loss/production mechanisms. This is carried out by following the method described by ${ }^{(11)}$.

In order to substantiate the presence of HF Doppler oscillations, we have looked into the surface magnetic field measurements too. The geographic coordinates of ground based magnetometers presented in this paper are given in the Table 1. The magnetometer data at Kevo (KEV), Masi (MAS), Kilpisjarvi (KIL), Ivalo (IVA), Muonio (MUO), Pello (PEL), Pondichery $(\mathrm{PON})$, Tirunelveli (TIR) are part of magnetic observatories of the IMAGEmagnetometer network andIndian Institute of Geomagnetism. It is to be noted that the time resolution of all the ground magnetometer data is 1 minute. 
Table 1. Geographic coordinates of the Magnetometer and HF Doppler stations

\begin{tabular}{lll}
\hline Magnetometer Stations & Longitude & Latitude \\
\hline Kevo & 69.76 & 27.01 \\
Masi & 69.46 & 23.70 \\
Kilpisjarvi & 69.02 & 20.79 \\
Ivalo & 68.56 & 27.29 \\
Muonio & 68.02 & 23.53 \\
Pello & 66.90 & 24.08 \\
\hline
\end{tabular}

\section{Results and Discussion}

HF Doppler oscillations of vertical velocity at $3.5 \mathrm{MHz}$ frequency on $14^{\text {th }}$ August 2003 are shown in the Figure 1 . This was a moderately disturbed day $(\mathrm{Ap}=14$, ,). The Doppler oscillations are found with a time period of nearly $150-600 \mathrm{~s}$ and with peak to peak amplitude of $8.5 \mathrm{~m} / \mathrm{s}$ to $35 \mathrm{~m} / \mathrm{s}$. The Doppler oscillations with period 300-600 s are very common at electrojet stations during daytime and post-sunset hours ${ }^{(12)}$. The observed oscillations are compared with geomagnetic field data from three high latitude stations, namely, Kevo (KEV), Masi (MAS) and Kilpisjarvi (KIL) for the local time interval 2000 LT to 2300 LT. The H component of magnetic field at these stations changed in the same manner, although there are some variations in the amplitude. For 2120 LT to 2220 LT, Pc5 geomagnetic field oscillations of about 10 min with amplitude 150 to $200 \mathrm{nT}$ was detectable at stations KEV, MAS and KIL. Thus, the magnetic field oscillations of other stations under IMAGE magnetometer network show similar variations. The HFD oscillations observed are found to be in phase with the geomagnetic field oscillations of high latitude origin. The periodicity of HFD oscillations observed is nearly $15 \mathrm{~min}$ or slightly greater than this value. From $2120 \mathrm{LT}$ to around $2130 \mathrm{LT}$ the geomagnetic field is more southward compared to other time intervals. After $2130 \mathrm{LT}$, it turns to more northward. This wave behaviour is clearly reflected in the HFD oscillations. The H component variations are clearly visible rather than $\mathrm{D}$ component of magnetic field.

The top panel of Figure 2 shows the temporal variation of $\mathrm{H}$ component of geomagnetic field at the high latitude stations viz. Ivalo (IVA), Muonio (MUO) and Pello (PEL) during the time interval 1700 LT to 2000LT on 15 May 2005. This day was a geomagnetically disturbed day $(\mathrm{Ap}=108)$ ). No magnetic field data were available at Tirunelveli and Pondicherry on that day. Similar to the event observed on $14^{\text {th }}$ August 2003, geomagnetic oscillations of higher amplitudes are found at 1700 LT to 1830 LT. After this interval, the amplitude of magnetic field oscillations decreases and finally dies out. Coherent and simultaneous geomagnetic field oscillations at Ivalo, Muonio and Pello in the evening time can be clearly visible in the $\mathrm{H}$ component than in the D component. The bottom panel of Figure 2 represents the HFD oscillations in the vertical velocity at $3.5 \mathrm{MHz}$ which was clearly observable at Thiruvananthapuram during the interval 1700-2000 LT. It is very clear that the HFD oscillations at Thiruvananthapuram varied in the same way as the high latitude long-period geomagnetic field oscillations at above said stations and are in the same phase.

The observations from the Figure 1 suggest that the observed features are long period HFD oscillations and could be due to the high latitude Pc5 pulsation activity. The amplitude of Pc5 oscillations are found to increase as we move from low latitude to high latitude. The observed HFD oscillations at Thiruvananthapuram correspond to $0.6 \mathrm{mV} / \mathrm{m}$ or less in the dawn-to-dusk electric field. It was quite evident that all positive and negative peaks of HFD variations have one to one correspondence and in phase with those of the Pc5 geomagnetic field oscillations. The HF Doppler data and H component are sampled every minute. ${ }^{(13,14)}$ argued that the amplitude of geomagnetic field oscillations decreases with latitude but their periodicity is unaffected by latitude or longitude. But the magnetic $\mathrm{H}$ component at Pondicherry and Tirunelveli did not show corresponding oscillations on 14 August 2003. So we conclude that present HFD oscillations are not due to the incoming hydromagnetic waves directly to low and equatorial latitudes. Thus the HFD oscillations coherent with high latitude geomagnetic field oscillations are primarily believed to be due to the prompt penetration of high latitude convection electric field. The simultaneous occurrence of geomagnetic field oscillations at the day time equator and the high latitudes can be interpreted as the instantaneous transmission of polar electric field ${ }^{(3,14)}$.

It must be noted that magnetic $\mathrm{H}$ component variations at Kevo, Masi and Kilpisjarvi on 14 April 2003 during the interval 2000 LT 2300 LT show one to one correspondence with the HF Doppler oscillations. In this event too, the observed HFD oscillations are believed to be due to the ExB drifts caused by the penetrating electric field. The dawn-to-dusk polar electric field causes a positive deflection in the night time HF Doppler frequency at equatorial latitudes. It is evident from the above discussion that, long period HFD oscillations $(15 \mathrm{~min}$.) are plausible in the equatorial latitudes coherent with geomagnetic disturbances originating from high latitudes. In fact, ${ }^{(15)}$ have reported similar long period range (6-14 min.) oscillations concomitantly over 
polar and equatorial latitudes and suggested that such oscillations can be observed much amplified over the equator due to Cowling conductivity. Furthermore, the presence of oscillatory twin vortex current system in the polar region Indicates that the high-latitude electric field associated with the long-period geomagnetic field oscillations is quite large scale with the dawndusk polarity, like the SC and DP2 fluctuation sudden expansion of the magnetosphere causes a dawn-to-dusk electric field at the polar ionosphere by a pair of field aligned currents (FAC) ${ }^{(16)}$. The morning twin vortex current system extended to lower latitudes in turn can produce a westward electric field at the dip equator on the night side ${ }^{(9,17)}$.

The observed HF Doppler oscillations over equator on 14 August 2003 and 15 May 2005 clearly indicate that long period geomagnetic fluctuations can instantaneously reflect over equator. Although these observations are explained primarily on the basis of current understanding of magnetosphere-ionosphere coupling during disturbed periods, more comprehensive observations in tandem with modelling are needed to arrive a cause a and effect relationship. Nevertheless, we believe that the present observations are important and can have significant implications in the understanding of space weather and its effects over the solar terrestrial environment in detail.

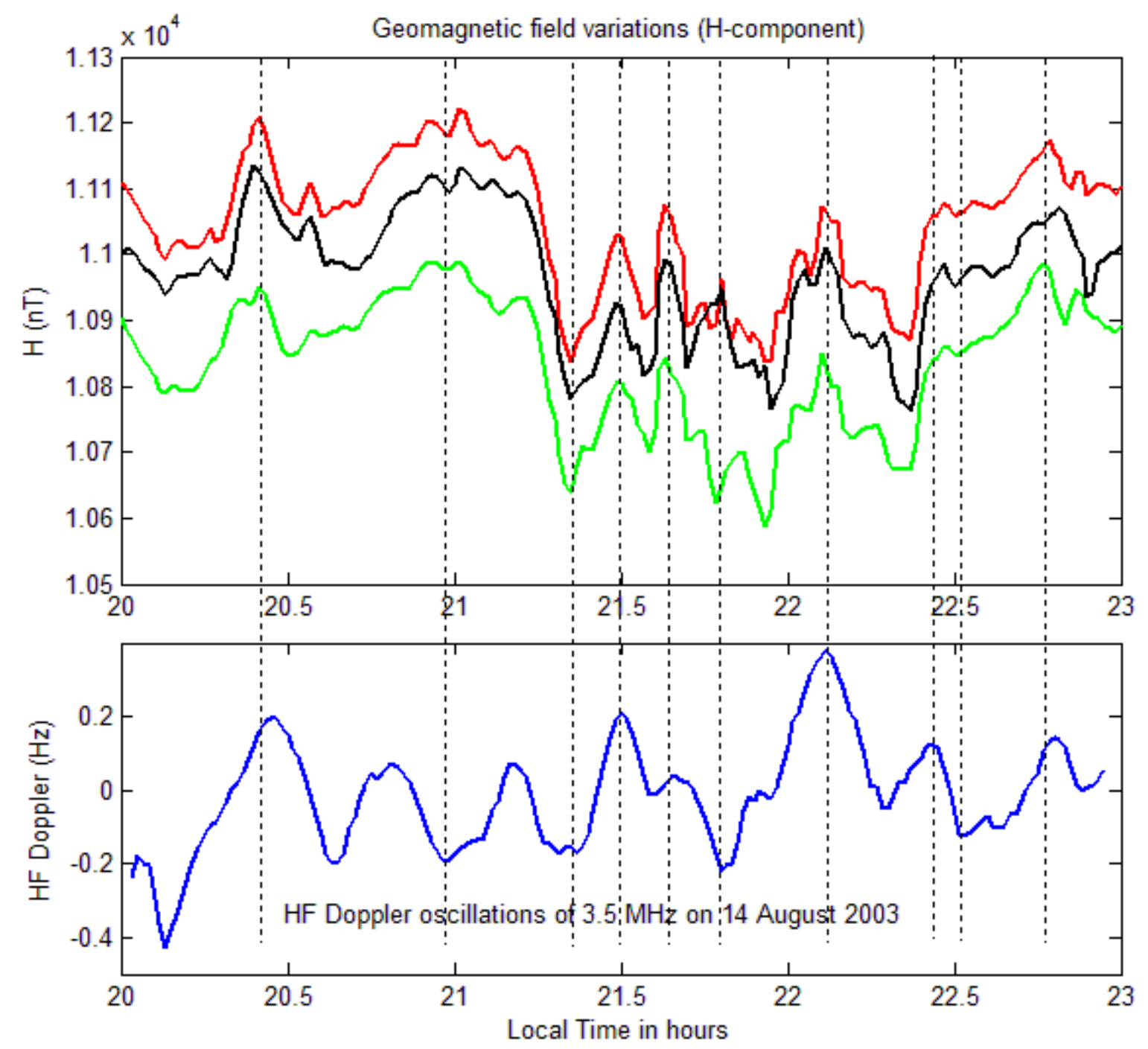

Fig 1. Magnetic H component variations observed at three high latitude stations Kevo, Masi and Kilpisjarvi for the interval of 2000LT 2300LT on 14 April 2003 (top). Doppler frequency oscillation at $3.5 \mathrm{MHz}$ observed at nighttime low latitude station Trivandrum $\left(8.33^{\circ} \mathrm{N}, 77^{\circ} \mathrm{E}, \operatorname{dip}\right.$ $0.4^{\circ} \mathrm{N}$ ) for the same interval (bottom). 


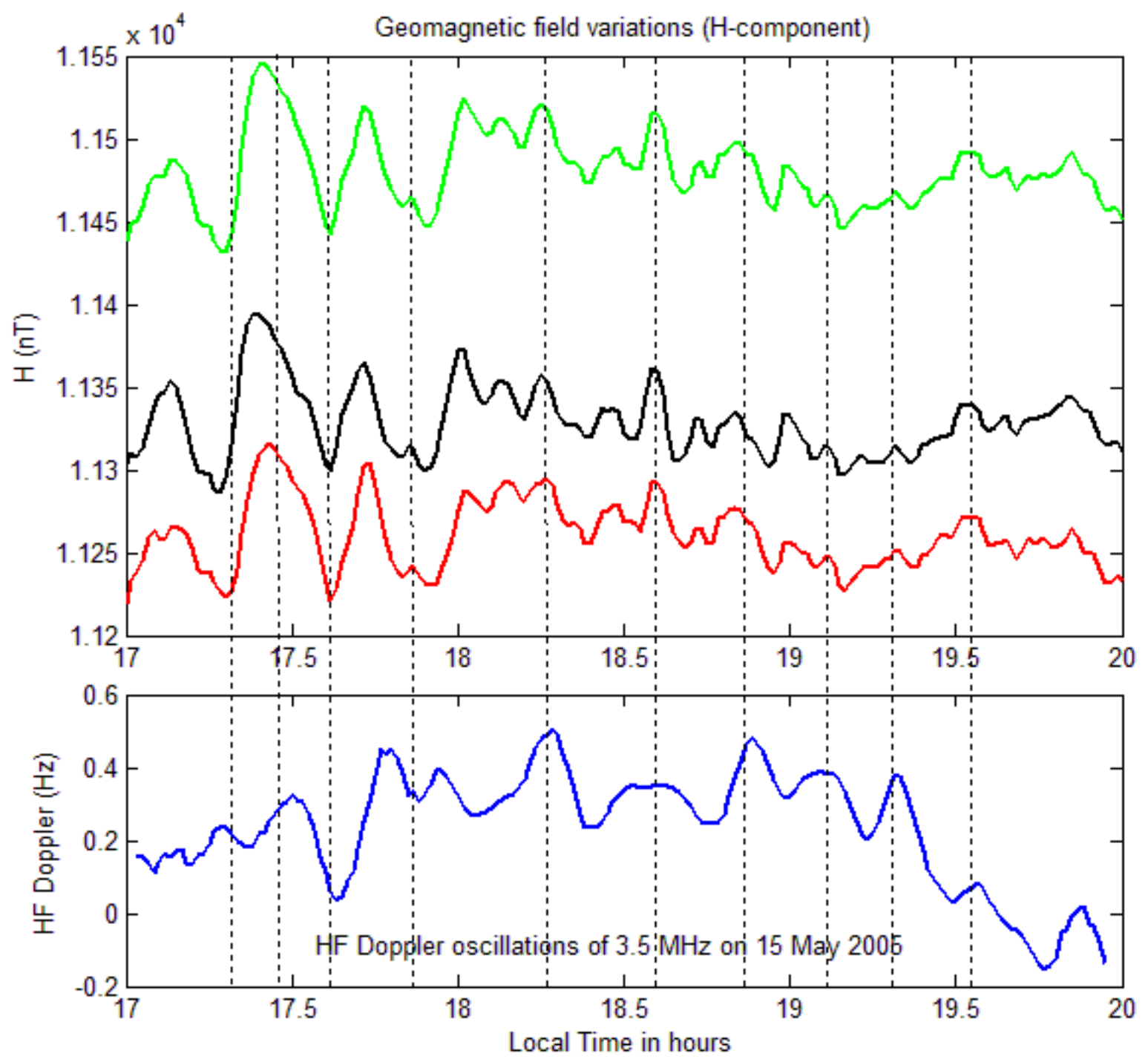

Fig 2. Magnetic H component variations observed at three high latitude stations Ivalo, Muonio and Pello for the interval of 1700LT 2000LT on 15 May 2005.(top) Doppler frequency oscillation at $3.5 \mathrm{MHz}$ observed at nighttime low latitude station Thiruvananthapuram $\left(8.33^{\circ} \mathrm{N}\right.$, $77^{\circ} \mathrm{E}$, dip $0.4^{\circ} \mathrm{N}$ ) for the same interval (bottom).

\section{Concluding remarks}

The Doppler velocity oscillations over Trivandrum around sunset time experimentally proved that they are due to prompt penetration of polar electric field and the magnetic field effects of which on the ground are identified as Pc5 micro-pulsations. The Doppler oscillations in the vertical plasma drift are due to the zonal component of time varying electric field in association with geomagnetic pulsations. The high latitude magnetic field oscillations in the evening nighttime sector are significantly affected by ionospheric currents which are strongly controlled by time dependent ionospheric conductivity. Thus, the results indicate that the HFD oscillations are driven by the simultaneous transmission of polar originated electric field. The polarity of electric field derived from HFD oscillations at nighttime equatorial latitude is in the same phase with the electric field derived from magnetic field oscillations. Thus, it can be concluded that, the HFD oscillations are primarily due to the ExB effects caused by the direct penetration of polar dawn-to-dusk electric field which causes geomagnetic field pulsations in the dip equatorial region. 


\section{References}

1) Simi KG, Susanth SG, Sumod SG. On the vector plasma drift measurements over Equatorial Ionosphere using HF Doppler radar - A brief review. Indian Journal of Science and Technology. 2020;13(35):3642-3651. Available from: https://dx.doi.org/10.17485/ijst/v13i35.1084.

2) Rishbeth H, Garriott OK. Relationship between simultaneous geomagnetic and ionspheric oscillations. Journal of Research of the National Bureau of Standards, Section D: Radio Science. 1964;68D(3). Available from: https://dx.doi.org/10.6028/jres.068d.051.

3) Kikuchi T, Araki T. Horizontal transmission of the polar electric field to the equator. Journal of Atmospheric and Terrestrial Physics. 1979;41(9):927-936. Available from: https://dx.doi.org/10.1016/0021-9169(79)90094-1.

4) Liu JY, Huang YN, Berkey FT. The phase relationship between ULF geomagnetic pulsations and HF Doppler frequency shift oscillations on March 24. $J$ Geomagnetism and Geoelectricity. 1991;45:109-114. Available from: https://doi.org/10.5636/jgg.45.109.

5) Reddy CA, Ravindran S, Viswanathan KS, Murthy BVK, Rao DRK, Araki T. Observations of Pc5 micropulsation-related electric field oscillations in the equatorial ionosphere. Annales Geophysicae. 1994;12(6):565-573. Available from: https://dx.doi.org/10.1007/s00585-994-0565-7.

6) Araki T. Global structure of geomagnetic sudden commencements. Planetary and Space Sciences. 1976;25:373-384. Available from: https://doi.org/10. 1016/0032-0633(77)90053-8.

7) Nayar SRP, Mathew TJ, Sreehari CV, Sumod SG, Devasia CV, Ravindran S, et al. Electrodynamics of the equatorial F-region ionosphere during pre-sunrise period. Annales Geophysicae. 2009;27(1):107-111. Available from: https://dx.doi.org/10.5194/angeo-27-107-2009.

8) Sumod SG, Pant TK, Nayar SRP. On the variability of the observed HF Doppler derived equatorial F-region plasma drifts during evening and morning hours and the chemical corrections therein. Ind J Radio And Space Phys. 2012;41:130-140. Available from: http://hdl.handle.net/123456789/14040.

9) Fejer BG, Gonzales CA, Farley DT, Kelley MC, Woodman RF. Equatorial electric fields during magnetically disturbed conditions 1 . The effect of the interplanetary magnetic field. Journal of Geophysical Research. 1979;84(A10). Available from: https://dx.doi.org/10.1029/ja084ia10p05797.

10) George T, Joymon D, Solomon S, Rajesh P, Nayar S, Revathy K. Fluctuations in vertical plasma drift and magnetic activity. J Atmos Terr Phys. 1998;27:233235. Available from: http://nopr.niscair.res.in/handle/123456789/35314.

11) Nayar SR, Bhuvanendran C, Devasia JN, Subbarao CV, V KS. Meridional wind derived from HF doppler radar and ionosonde over the magnetic equator. Ind J Radio And Space Phys. 2004;33:367-372. Available from: http://hdl.handle.net/123456789/19341.

12) Sastri JH, Ramesh KB, Rao DRK, Rao JVSV. Oscillations in F-region Doppler velocity in the dip equatorial region associated with ULF geomagnetic pulsations. Journal of Atmospheric and Terrestrial Physics. 1993;55(9):1271-1280. Available from: https://dx.doi.org/10.1016/0021-9169(93)90051-y.

13) Ziesolleck CW, Chamalaun FH. Seasonal A two-dimensional array study of low-latitude PC 5 geomagnetic pulsations. Journal of Geophysical Research. 1993;98(A8):13703-13713. Available from: https://doi.org/10.1029/93JA00637.

14) Nayar SRP. Investigation of height gradient in vertical plasma drift at equatorial ionosphere using multifrequency HF Doppler radar. Journal of Geophysical Research. 2004;109(A12). Available from: https://dx.doi.org/10.1029/2004ja010641.

15) Motoba T, Kikuchi T, Okuzawa T, Yumoto K. Dynamical response of the magnetosphere-ionosphere system to a solar wind dynamic pressure oscillation. Journal of Geophysical Research: Space Physics. 2003;108(A5):1206-1219. Available from: https://dx.doi.org/10.1029/2002ja009696.

16) Motoba T, Kikuchi T, Lühr H, Tachihara H, Kitamura TI, Hayashi K, et al. Global Pc5 caused by aDP2-type ionospheric current system. Journal of Geophysical Research: Space Physics. 2002;107(A2):1032-1047. Available from: https://dx.doi.org/10.1029/2001ja900156.

17) Sastri JH, Varma VKM, Nayar SRP. Height gradient of F region vertical drift in the evening equatorial ionosphere. Geophysical Research Letters. 1995;22(19):2645-2648. Available from: https://dx.doi.org/10.1029/95gl02668. 